\title{
Stable expression of small interfering RNA sensitizes TEL-PDGF $\beta R$ to inhibition with imatinib or rapamycin
}

\author{
Jing Chen, ${ }^{1}$ Nathan R. Wall, ${ }^{2}$ Kerry Kocher, ${ }^{1}$ Nicole Duclos, ${ }^{1}$ Doriano Fabbro, ${ }^{3}$ Donna Neuberg, ${ }^{4}$ \\ James D. Griffin, ${ }^{4}$ Yang Shi, ${ }^{2}$ and D. Gary Gilliland ${ }^{1}$
}

\begin{abstract}
${ }^{1}$ Howard Hughes Medical Institute, Division of Hematology, Brigham and Women's Hospital and Harvard Medical School, ${ }^{2}$ Department of Pathology, Harvard Medical School, Boston, Massachusetts, USA. ${ }^{3}$ Novartis Pharma AG, Basel, Switzerland. ${ }^{4}$ Dana-Farber Cancer Institute, Boston, Massachusetts, USA.
\end{abstract}

\begin{abstract}
Small molecule inhibitors, such as imatinib, are effective therapies for tyrosine kinase fusions BCR-ABLTEL-PDGF $\beta R$-mediated human leukemias, but resistance may develop. The unique fusion junctions of these molecules are attractive candidates for molecularly targeted therapeutic intervention using RNA interference (RNAi), which is mediated by small interfering RNA (siRNA). We developed a retroviral system for stable expression of siRNA directed to the unique fusion junction sequence of TEL-PDGF $\beta R$ in transformed hematopoietic cells. Stable expression of the siRNA resulted in approximately $90 \%$ inhibition of TEL-PDGF $\beta$ R expression and its downstream effectors, including PI3K and mammalian target of rapamycin (mTOR). Expression of TEL-PDGF $\beta$ R-specific siRNA (TPsiRNA) significantly attenuated the proliferation of TEL-PDGF $\beta \mathrm{R}$-transformed $\mathrm{Ba} / \mathrm{F} 3$ cells or disease latency and penetrance in mice induced by intravenous injection of these $\mathrm{Ba} / \mathrm{F} 3$ cells. Although a $90 \%$ reduction in TEL-PDGF $\beta \mathrm{R}$ expression was insufficient to induce cell death, stable siRNA expression sensitized transformed cells to the PDGF $\beta R$ inhibitor imatinib or to the mTOR inhibitor rapamycin. TPsiRNA also inhibited an imatinib-resistant TEL-PDGF $\beta$ R mutant, and the inhibition was enhanced by siRNA in combination with PKC412, another PDGF $\beta$ R inhibitor. Although siRNA delivery in vivo is a challenging problem, stable expression of siRNA, which targets oncogenic fusion genes, may potentiate the effects of conventional therapy for hematologic malignancies.
\end{abstract}

\section{Introduction}

Tyrosine kinase fusions, including BCR-ABL and TEL-PDGF $\beta$ R, are well-validated therapeutic targets in human leukemias (1). Small molecule inhibitors, such as imatinib, are effective therapies for BCR-ABL- and TEL-PDGF $\beta$ R-mediated leukemias. Imatinib is a selective inhibitor of tyrosine kinases, including ABL, PDGF $\beta R$, PDGFR $\alpha$, ABL-related gene (ARG), and human cellular homolog of v-kit Hardy-Zuckerman 4 feline sarcoma viral oncogene (c-KIT) (2). Imatinib has been demonstrated to be safe and effective in the clinical treatment of BCR-ABL-positive chronic myelogenous leukemia (CML) and TEL-PDGF $\beta$ Rinduced chronic myelomonocytic leukemia (CMML), as well as in the treatment of other solid tumors such as gastrointestinal stromal cell tumors (GIST) associated with activating mutations of c-KIT (3-5). However, imatinib is not curative as a single agent, and clinical resistance may develop. Drug-resistance point mutations in the ABL kinase domain of BCR-ABL and overexpression of BCR-ABL have been identified in patients who relapsed while on imatinib therapy $(2,6-9)$. Therefore, the development of alternative molecularly targeted therapies that

Nonstandard abbreviations used: anaplastic lymphoma kinase (ALK); chronic myelogenous leukemia (CML); chronic myelomonocytic leukemia (CMML); FMS-like receptor tyrosine kinase 3 (FLT3); human cellular homolog of v-kit Hardy-Zuckerman 4 feline sarcoma viral oncogene (c-KIT); insulin-like growth factor receptor (IGFR); long tandem repeat (LTR); mammalian target of rapamycin (mTOR); nucleophosmin (NPM); phospholipase C- $\gamma$ (PLC $\gamma$ ); RNA interference (RNAi); small interfering RNA (siRNA); signal transducer and activator of transcription (STAT); TEL-PDGF $\beta$ Rspecific siRNA (TPsiRNA)

Conflict of interest: The authors have declared that no conflict of interest exists.

Citation for this article: J. Clin. Invest. 113:1784-1791 (2004).

doi:10.1172/JCI200420673. might enhance specificity and clinical efficacy, as well as overcome the problem of resistance, is of great clinical interest.

Chromosomal translocations are recurrent findings in leukemias and some solid tumors, and they frequently result in abnormal expression of chimeric fusion oncoproteins (1). The unique fusion junctions of these molecules are attractive candidates for molecularly targeted therapeutic intervention using RNA interference (RNAi). RNAi is an evolutionarily conserved process of posttranscriptional suppression of gene expression and is mediated by 21- to 23-nucleotide (nt) sequence-specific doublestranded small interfering RNA (siRNA) in mammalian cells without initiating the nonspecific double-stranded RNA responses (10-12). Chemically synthesized siRNAs targeting the BCR-ABL fusion junction have been tested for reduction in BCR-ABL protein expression in leukemia cells (13-15). However, the limitation of such an oligonucleotide-based system is that the delivery of siRNA to mammalian cells largely depends on transfection efficiency, and for many hematopoietic cell lines as well as primary cell lines, efficient transfection cannot be achieved. Moreover, transfected siRNA cannot induce RNAi over a long-term period. One solution is to express the specific siRNA molecules in cells. The intracellular transcription of siRNAs has been achieved by using pol III-dependent promoters, such as the small nuclear RNA (snRNA) U6 promoter and the human RNAse P RNA H1 promoter, to drive expression of short hairpin RNAs that are processed into siRNAs in mammalian cells (16-19).

The TEL-PDGF $\beta R$ fusion was identified as a consequence of $\mathrm{t}(5 ; 12)$ (q33; p13) chromosomal translocation, a recurring cytogenetic abnormality associated with CMML that is characterized by abnormal myelopoiesis with eosinophilia, myelofibrosis, and fre- 
quent progression to acute myeloid leukemia (20). Imatinib inhibits the growth of cells expressing TEL-PDGFR fusion protein and has been demonstrated to be effective in the clinical treatment of CMML induced by TEL-PDGF $\beta R(5,21)$. As presented in this report, we have developed a retroviral system for stable expression of siRNA directed to the unique junction sequences of fusion tyrosine kinases in transformed hematopoietic cells. We investigated the utility of siRNAs alone or in combination with small molecule inhibitors to impair TEL-PDGFßR-mediated hematopoietic transformation. Moreover, an inhibitory siRNA-based strategy was useful to overcome the resistance of a TEL-PDGF $\beta$ R mutant to imatinib.

\section{Methods}

Vectors. The human pol III promoter $\mathrm{H} 1$ was cloned from genomic DNA by PCR and inserted into pBluescript II as described to make pSUPER (22). The oligonucleotides encoding the TEL-PDGF $\beta R$ siRNA (TPsiRNA) were 5'-GATCCCCTGAAGAAGC CTTGCCСTTTTTCAAGAGAAAAGGGCAAGGCTTCTTCATTTTTGGAAA-3' and 5'-AGCTTTTCCAAAAATGAAGAAGCCTTGCССТTTTCTCTTGAAAAAGGGCAAGGCTTCTTCAGGG-3'. These oligonucleotides were annealed and subcloned to downstream of the H1 promoter in pSUPER by using HindIII and BglII. The resulting H1_TPsiRNA cassette was subcloned into $3^{\prime} \Delta$ long tandem repeat ( $\triangle \mathrm{LTR}$ ) of $\mathrm{PQCXIP}$ vector (BD Biosciences Clontech, Palo Alto, California, USA) by using NheI to generate pQCXIP_ H1_TPsiRNA. The H1 promoter was also subcloned into $3^{\prime}$ LTR pQCXIP by using NheI with the introduction of XhoI and HpaI sites following $\mathrm{H} 1$. The oligonucleotides encoding the NPM-ALK siRNA were 5'-TCGACCCATGAAGAAGCCTTGCCCTTTTCAAGAGAAAGGGCAAGGCTTCTTCATTTTTTGGAAAGTT-3' and 5'AACTTTCCAAAAAATGAAGAAGCCTTGCCCTTTCTCTTGAAAAGGGCAAGGCTTCTTCATGGG-3'. These oligonucleotides were annealed and subcloned to downstream of the $\mathrm{H} 1$ promoter in pQCXIP by using XhoI and HpaI to generate pQCXIP_H1_NAsiRNA. Mutation T681I was introduced into TEL-PDGF $\beta$ R by using the QuikChange-XL site-directed mutagenesis kit according to the manufacturer's instructions (Stratagene, La Jolla, California, USA) using pMSCV-neoEB-TEL-PDGF $\beta R$ as a template. The primer used was 5'-GGAGGACCCATCTATATCATCATTGAGTACTGCCGCTACGGAGAC-3'.

$\mathrm{Ba} / \mathrm{F} 3$ cell culture and retroviral transduction. Ba/F3 cells were cultured in RPMI 1640 medium with 10\% FBS and $1.0 \mathrm{ng} / \mathrm{ml} \mathrm{IL-3.}$ The 293T cells used to generate the siRNA retroviral supernatant were cultured in DMEM with $10 \%$ FBS. To generate Ba/F3 cell lines stably expressing siRNA, $1 \times 10^{6}$ TEL-PDGF $\beta$ R or nucleophosmin-anaplastic lymphoma kinase (NPM-ALK) neomycin-resistant stable $\mathrm{Ba} / \mathrm{F} 3$ cells were transduced with retroviral supernatant carrying PQCXIP_H1_TPsiRNA or pQCXIP_H1_NAsiRNA, respectively, in the presence of Polybrene $(10 \mu \mathrm{g} / \mathrm{ml})$ and cultured for 48 hours. The cells were then selected with $1.0 \mathrm{mg} / \mathrm{ml} \mathrm{G} 418$ and 1.0 $\mathrm{ng} / \mathrm{ml}$ puromycin in the presence of IL-3 for another 8-10 days. To assess IL-3-independent outgrowth, Ba/F3 cells were washed three times with RPMI and cultured in RPMI with 10\% FBS without IL-3. The number of live cells was determined by trypan blue staining and was scored daily. For cell viability assay, $1 \times 10^{5} \mathrm{Ba} / \mathrm{F} 3$ cells were cultured in 24-well plates under the condition of IL-3 withdrawal and, in some instances, chemotherapeutic drugs. The number of viable cells at each experimental time point was determined by using the Celltiter $96 \mathrm{AQ}_{\text {uesous }}$ one solution proliferation kit (Promega Corp., Madison, Wisconsin, USA).
Mice. Athymic nude mice were each injected with $1 \times 10^{6} \mathrm{Ba} / \mathrm{F} 3$ cells stably expressing TEL-PDGF $\beta$ R with or without TPsiRNA, or $\mathrm{Ba} / \mathrm{F} 3$ cells transduced with empty vector as control, via marginal tail vein ( $n=4$ for each $\mathrm{Ba} / \mathrm{F} 3$ cell line). In a second study, syngeneic Balb/C mice were injected with variant $\mathrm{Ba} / \mathrm{F} 3$ cells $(n=4$ for empty vector and TEL-PDGF $\beta$ R control cell lines; $n=8$ for cells stably expressing TEL-PDGF $\beta$ R and TPsiRNA). The log rank test was used to assess statistical significance for differences in survival.

Western blotting analysis. To assay for the phosphorylation level of various different proteins, $\mathrm{Ba} / \mathrm{F} 3$ cells were treated with serum starvation and in some instances imatinib at increasing concentrations for 4 hours prior to lysis. The cell extracts were applied to immunoprecipitation with various antibodies and the protein G-Sepharose (Amersham Pharmacia, Piscataway, New Jersey, USA). Immunocomplexes were resolved on SDS/PAGE (12.5\%) for Western blotting. Antibodies used included anti-PDGF $\beta$ R tail serum (BD Biosciences Pharmingen, San Diego, California, USA); anti-PI3K (p85) antiserum, 4G10 anti-phosphotyrosine antibody (Upstate Biotechnology, Lake Placid, New York, USA); antibodies recognizing STAT5b and phospho-PI3K p85 (Tyr-508) (Santa Cruz Biotechnology, Santa Cruz, California, USA); phospho-STAT5 (Tyr-694), STAT3, phospho-STAT3 (Tyr-705), phospholipase C (PLC $\gamma$ ) and phosphor-PLC $\gamma$ (Tyr-783) (Cell Signaling Technology, Beverly, Massachusetts, USA).

RT-PCR and Northern blotting. Whole RNA was isolated from $\mathrm{Ba} /$ F3 cells using the RNeasy Mini Kit (QIAGEN, Valencia, California, USA). Primers designed to specifically amplify the TEL-PDGF $\beta$ R breakpoint region are 5'-CAGCCGGAGGTCATACTGG-3' and 5'GCATGATGAGGATGATAAGGG-3'. The RT-PCR reactions were performed using QuantiTect SYBR Green RT-PCR kit (QIAGEN) on an iCycler iQ Multi-Color Real Time PCR Detection system (Bio-Rad Laboratories, Hercules, California, USA). TEL-PDGF $\beta R$ mRNA level was normalized against endogenous GAPDH mRNA level. For Northern blotting, total RNA was isolated using TRIZOL Reagent (Invitrogen Life Technologies, Carlsbad, California, USA) from $\mathrm{Ba} / \mathrm{F} 3$ cells. RNA $(30 \mu \mathrm{g})$ was applied onto $12 \%$ (wt/vol) polyacrylamide-urea gels and transferred by electroblotting onto ZetaProbe GT nylon membranes (Bio-Rad Laboratories). Membranes were prehybridized and hybridized using ExpressHyb Hybridization Solution (BD Biosciences Clontech) following manufacturer's instructions. TPsiRNA was probed by ${ }^{32} \mathrm{P}$-labeled antisense oligonucleotides (5'-TGAAGAAGCCTTGCCCTTT-3') for 1 hour at $42^{\circ} \mathrm{C}$ and visualized by autoradiography.

\section{Results}

Retroviral delivery of siRNA directed against TEL-PDGF $\beta R$ breakpoint into hematopoietic cells. To target the unique fusion junction sequence in TEL-PDGF $\beta R$, a 19-bp stem-loop structure was designed as described previously for siRNA expression (22), including a 19-nt sense strand of target sequence that is derived from the breakpoint of the TELPDGF $\beta R$ gene and a short spacer sequence that is followed by a reverse complement of the same 19-nt sequence (Figure 1A). We cloned the human pol III-dependent RNAse P RNA H1 promoter from genomic DNA and subcloned the $\mathrm{H} 1$ promoter followed by the siRNA hairpin cassette into the $3^{\prime}$ LTR of PQCXIP, a self-inactivating (SIN) retroviral vector to minimize potential promoter interference from the viral $5^{\prime}$ LTR (Figure 1B). This strategy results in two copies of the H1-siRNA cassette after reverse transcription of the integrated provirus in the $5^{\prime}$ and $3^{\prime}$ LTR, respectively, which has been reported to generate higher levels of siRNA and enhanced inhibition of target genes (23). 
A
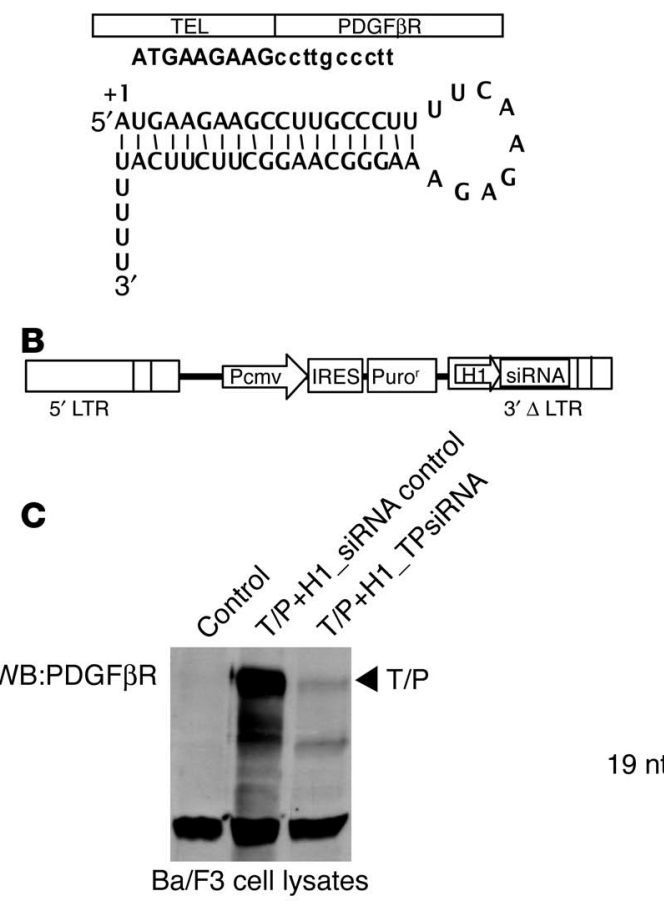

Figure 1

Retroviral delivery of siRNA directed against TEL-PDGF $\beta$ R (T/P) breakpoint into hematopoietic cells. (A) The upper panel shows a schematic representation of TEL-PDGF $\beta$ R fusion tyrosine kinase. The fusion junction that the siRNA was designed to target is indicated (upper and lower case letters correspond to TEL and PDGF $\beta$ R sequence, respectively). The lower panel shows the predicted short hairpin transcript with a $3^{\prime}-U_{5}$ pol III stop signal. (B) Schematic representation of pQCXIP_H1_TPsiRNA retroviral vector. The cassette of siRNA hairpin structure driven by pol III promoter $\mathrm{H} 1$ was subcloned into the $3^{\prime} \Delta$ LTR of the self-inactivating retroviral vector pQCXIP. $\Delta$ indicates deletion. Pcmv, CMV promoter; IRES, internal ribosomal entry site; Puro', puromycin-resistance; H1, pol III RNAse P RNA H1 promoter. (C) Expression of TEL-PDGF $\beta$ R in stably transformed Ba/F3 cells. pQCXIP_H1_TPsiRNA was introduced into TEL-PDGF $\beta$ R stable $\mathrm{Ba} / \mathrm{F} 3$ cells following retroviral transduction. TEL-PDGF $\beta R$ was detected with a rabbit polyclonal antibody recognizing PDGF $\beta R$ C-terminal tail. Ba/F3 cells transduced with an empty retroviral vector, as well as stable Ba/F3 cells expressing TEL-PDGF $\beta R$ transduced with $\mathrm{pQXCIP}$ retroviral vector encoding a nonspecific siRNA construct, were included as controls. WB, Western blot. (D) RT-PCR analysis with total RNA from different stable Ba/F3 cells was performed with a pair of primers amplifying approximately $350 \mathrm{bp}$ sequence overlapping the breakpoint of TEL-PDGF $\beta R$. The mRNA level of TEL-PDGF $\beta R$ was normalized to the endogenous GAPDH mRNA level as indicated. (E) Northern blot analysis with total RNA from different stable Ba/F3 cells was performed and probed with ${ }^{32} \mathrm{P}$-labeled antisense 19 -nt oligonucleotide for TPsiRNA. The control 5S-rRNA band was detected with ethidium bromide staining. $\mathrm{Ba} / \mathrm{F} 3$ cells transduced with empty retroviral vector were included as a control.

We first tested the effect of the expressed siRNA on TEL-PDGFßR expression in $\mathrm{Ba} / \mathrm{F} 3$ cells stably transduced with TEL-PDGF $\beta \mathrm{R}$. The retroviral vector (PQCXIP_H1_TPsiRNA) containing the cassette of H1-driven TPsiRNA and a puromycin resistance gene was introduced into Ba/F3 cells stably expressing TEL-PDGF $\beta$ R. Western blot analysis of cells selected for resistance to puromycin demonstrated suppression of TEL-PDGF $\beta$ R protein expression (Figure 1C). Real-time RT-PCR analysis showed an approximately 4,000-fold decrease in mRNA level of TEL-PDGF $\beta$ R (Figure 1D), but no decrease was detected in the mRNA level of native TEL or PDGF $\beta$ R by RT-PCR (data not shown) in the cells following retroviral transduction of pQCXIP_H1_TPsiRNA. These results demonstrate that TEL- PDGF $\beta$ R-derived siRNA is highly specific for suppression of the fusion tyrosine kinase. Furthermore, North- dependence. These findings correlated with a marked decrement, but not abrogation, in activation of downstream effectors of TEL-PDGF $\beta$ R including STAT5, PI3K, STAT3 or PLC $\gamma$, respectively (Figure 2, C-F).

To evaluate the potential therapeutic efficacy of TPsiRNA in vivo, we used a murine mouse model in which $1 \times 10^{6}$ TEL-PDGF $\beta$ Rtransformed $\mathrm{Ba} / \mathrm{F} 3$ cells or TEL-PDGF $\beta \mathrm{R}$-transformed $\mathrm{Ba} / \mathrm{F} 3$ cells coexpressing the TPsiRNA were injected into the tail vein of nude mice (Figure 2G). No disease was observed in the mice injected with control $\mathrm{Ba} / \mathrm{F} 3$ cells transduced with an empty retroviral vector. $\mathrm{Ba} / \mathrm{F} 3$ cells stably expressing TEL-PDGF $\beta$ R alone caused tumor development and death with a median latency of 24 days after injection. In contrast, expression of inhibitory siRNA in cells stably transformed by TEL-PDGF $\beta$ R resulted in a statistically significant prolongation in survival $(P=0.0002)$, with a median latency of 41 days. Similar 
A

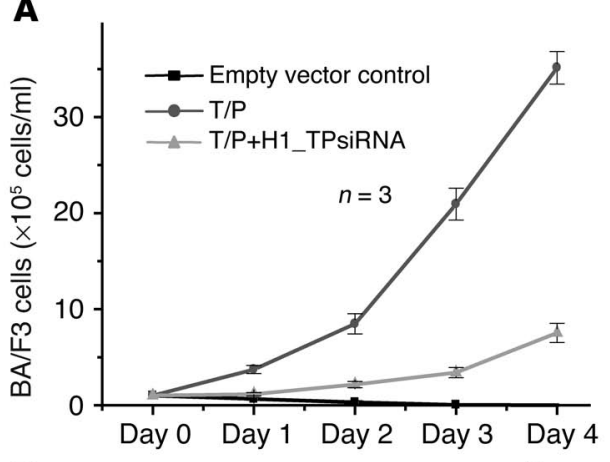

D

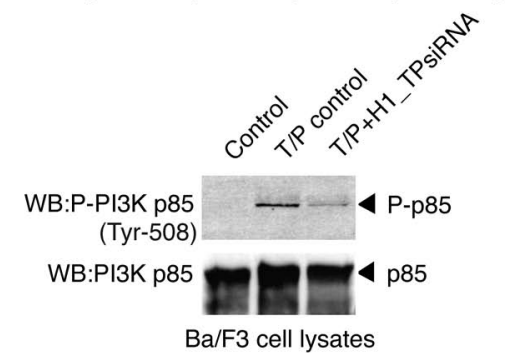

G

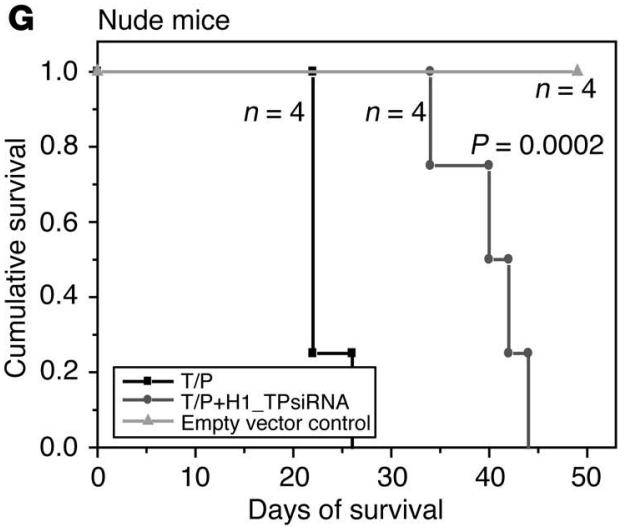

B

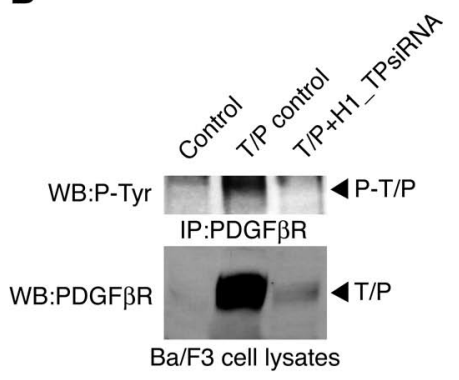

E

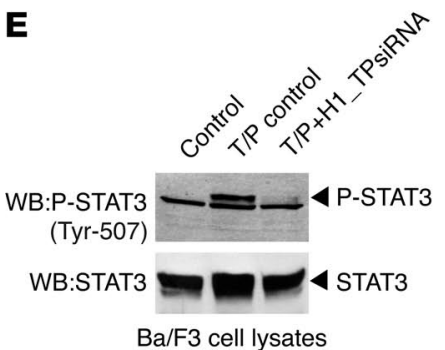

H

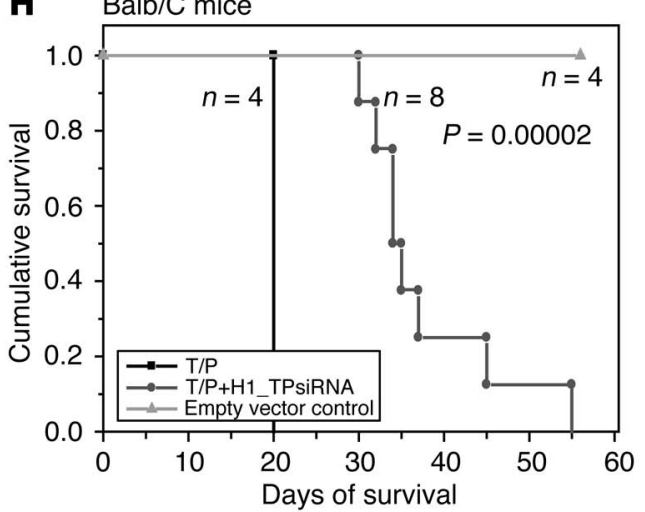

C

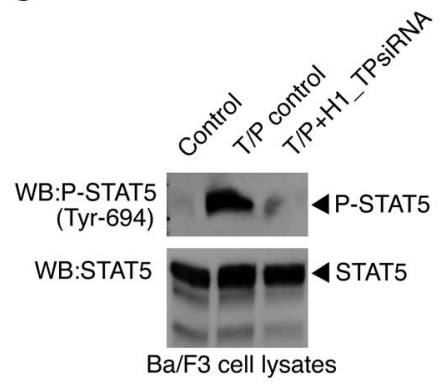

$\mathbf{F}$

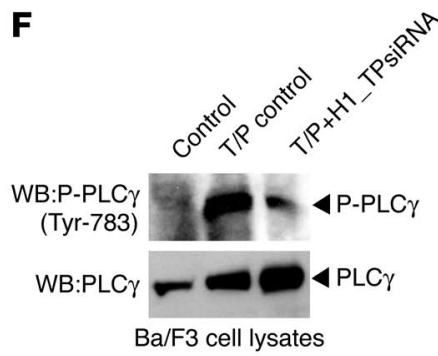

Figure 2

Stable siRNA expression inhibits TEL-PDGF $\beta$ R in vitro and in vivo. (A) Test for IL-3 independence of various TEL-PDGF $\beta R$ Ba/F3-derivative stable cell lines. G418-resistant Ba/F3 cells were treated with IL-3 withdrawal and counted daily. Ba/F3 cells transduced with empty retroviral vector were included as a control. (B) Autophosphorylation of TEL-PDGF $\beta$ R in Ba/F3 cells. TEL-PDGF $\beta$ R fusion was immunoprecipitated and probed with $4 \mathrm{G} 10$ anti-phosphotyrosine antibody. Ba/F3 cells transduced with empty retroviral vector were included as a control. The bottom panel shows TEL-PDGF $\beta$ R expression. P-Tyr, phosphor-tyrosine; P-T/P, phosphorylated-T/P. (C-F) Activation of STAT5, PI3K p85, STAT3 and PLC $\gamma$ by TEL-PDGF $\beta$ R was assessed with phospho-tyrosine-specific antibodies as described in Methods. (G and $\mathbf{H}$ ) Expression of TPsiRNA increases survival in murine models of leukemia mediated by TEL-PDGF $\beta$ R-transformed Ba/F3 cells. Nude mice (G) or Balb/C mice $(\mathbf{H})$ were injected with $\mathrm{Ba} / \mathrm{F3}$ cells stably transduced with empty vector control or TEL-PDGF $\beta R$, or TEL-PDGF $\beta R$ Ba/F3 cells coexpressing TPsiRNA. Kaplan-Meier survival plots are shown.

results were obtained in a second study in which $\mathrm{Ba} / \mathrm{F} 3$ cells were injected into syngeneic Balb/C mice. When compared with the control group injected with TEL-PDGF $\beta$ R stable cells, a significant increase in survival was observed in animals injected with TELPDGF $\beta$ R cells expressing siRNA $(P=0.00002$; Figure $2 \mathrm{H})$. These data indicate that TPsiRNA expression markedly attenuates the efficacy of TEL-PDGF $\beta \mathrm{R}$-mediated tumorigenesis in this murine allograft model. However, TPsiRNA expression alone in this context is not sufficient to abrogate TEL-PDGF $\beta$ R-induced transformation.

Stable siRNA expression sensitizes TEL-PDGF $\beta R$ for small molecule inbibitors and overcomes drug resistance. We next tested for possible synergistic effects between inhibitory siRNA and imatinib, a small molecule inhibitor of TEL-PDGF $\beta$ R tyrosine kinase activity. Imatinib inhibits the growth of TEL-PDGFR-transformed cells and is effective in the clinical treatment of CMML induced by TEL-PDGF $\beta R(5,21)$. Doseresponse curves demonstrated that TEL-PDGF $\beta \mathrm{R}$-transformed Ba/ F3 cells expressing the TPsiRNA were sensitized to imatinib in a cell viability assay (Figure $3 \mathrm{~A}$ ). For example, the $\mathrm{IC}_{50}$ of TEL-PDGF $\beta \mathrm{R}-$ transformed cells for imatinib was approximately $50 \mathrm{nM}$, whereas the $\mathrm{IC}_{50}$ for cells coexpressing the TPsiRNA was approximately 10 nM. Moreover, the Western blot results showed that stable expression of TPsiRNA sensitized TEL-PDGF $\beta$ R to inhibition by imatinib in autophosphorylation activity of TEL-PDGFßR, as well as activation of TEL-PDGF $\beta$ R downstream effectors including STAT5, PI3K or PLC $\gamma$ (Figure 3B). At high concentrations of imatinib (>100 nM), coexpression of TPsiRNA almost completely abrogated the kinase 


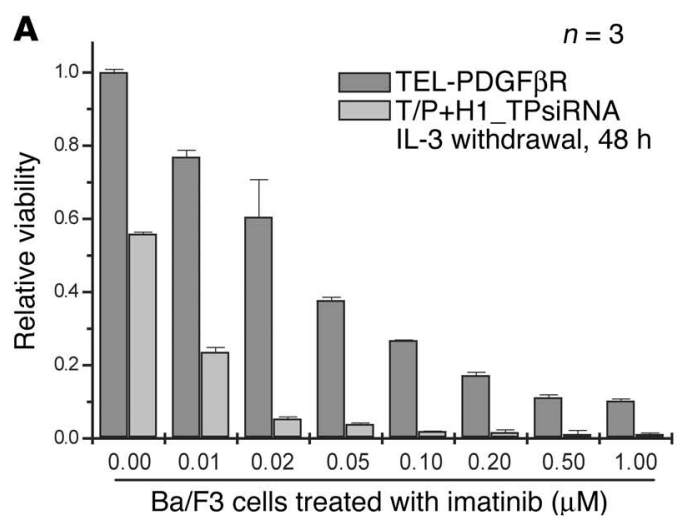

B

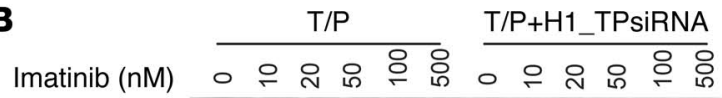

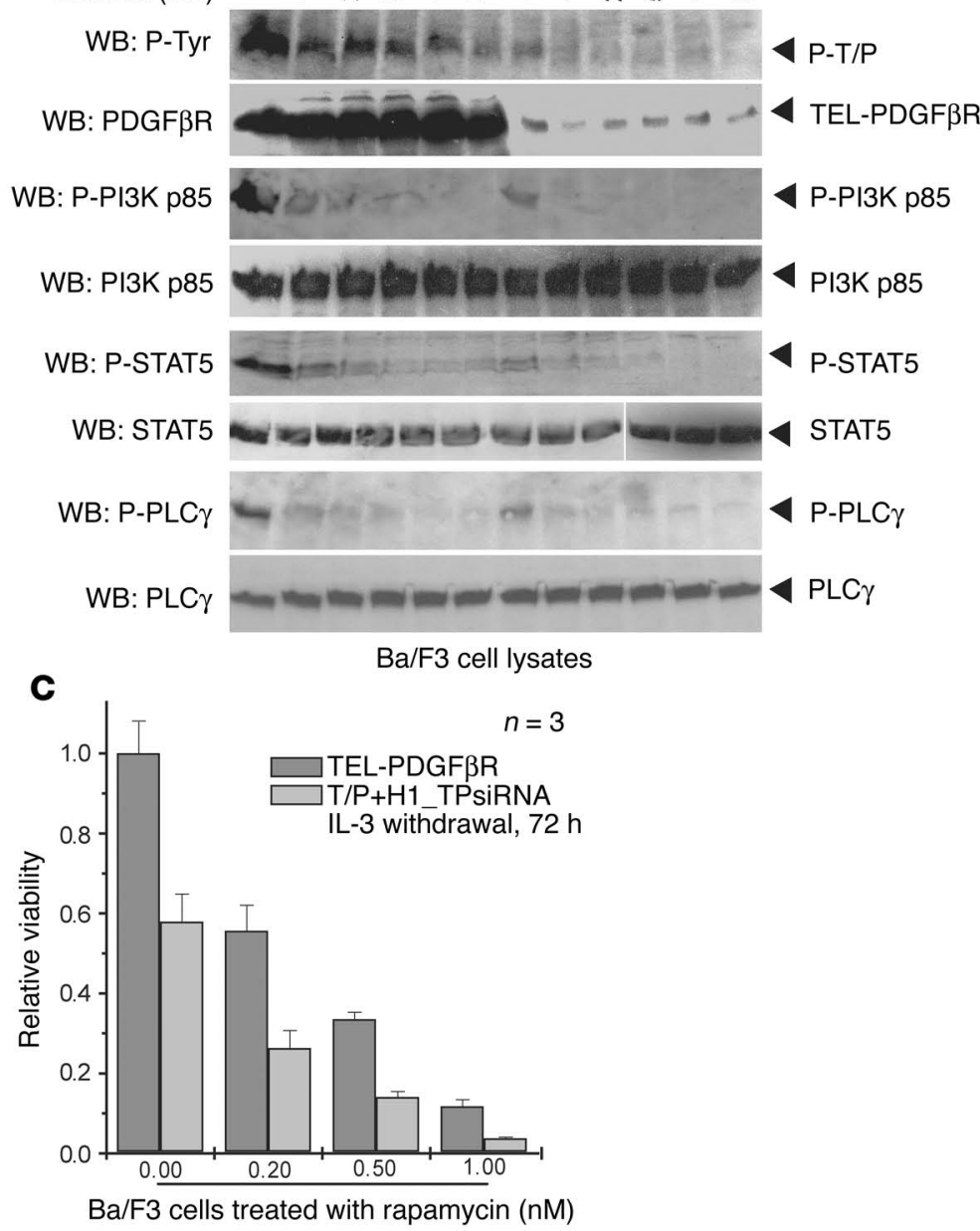

D

E
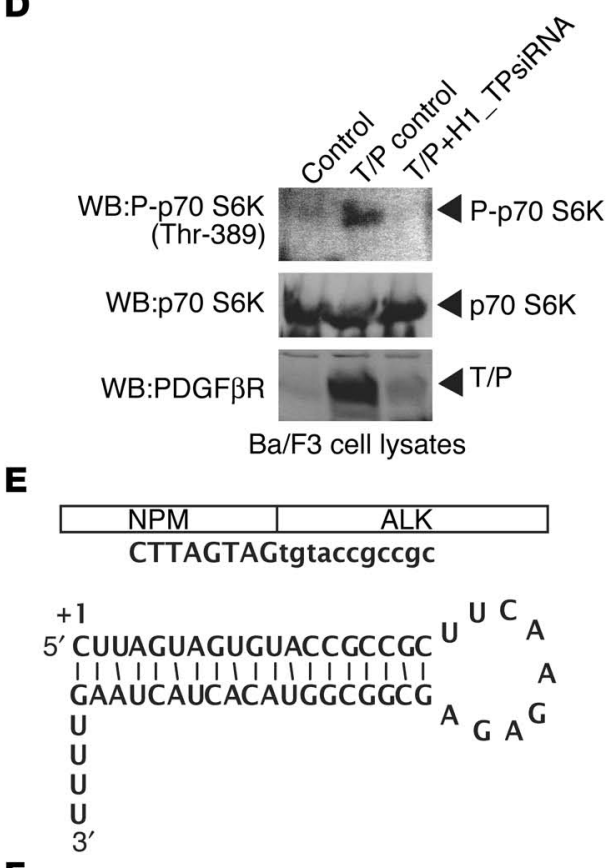

$\mathbf{F}$

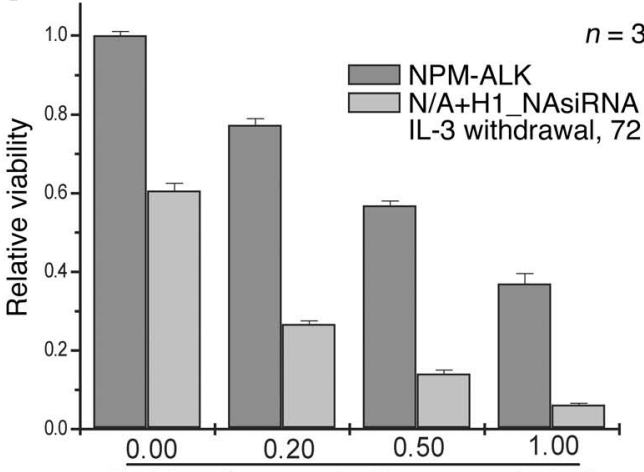

$\mathrm{Ba} / \mathrm{F} 3$ cells treated with rapamycin $(\mathrm{nM})$

G

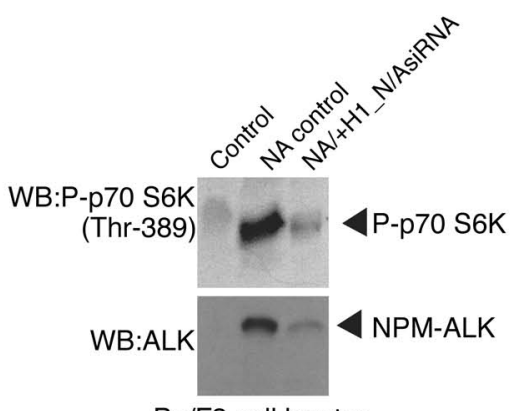

$\mathrm{Ba} / \mathrm{F3}$ cell lysates

\section{Figure 3}

Stable siRNA expression sensitizes TEL-PDGF $\beta$ R for small molecule inhibitors and overcomes drug resistance. (A) TPsiRNA expression sensitizes TEL-PDGF $\beta$ R-transformed Ba/F3 cells to inhibition by imatinib in a cell viability assay. The percentage of cell growth was normalized to the growth of cells in the absence of drug treatment. (B) TPsiRNA sensitizes TEL-PDGF $\beta$ R to inhibition by imatinib in auto-tyrosine phosphorylation and activation of TEL-PDGF $\beta$ R downstream signaling components. Western blot analysis was performed as described in Figure 2. (C) TPsiRNA sensitizes TEL-PDGF $\beta$ R-transformed Ba/F3 cells to the effects of mTOR inhibitor rapamycin in the cell viability assay. (D) Activation of mTOR assessed by phosphorylation of the mTOR target p70 S6 kinase. Ba/F3 cells transduced with empty retroviral vector were included as a control. (E) Schematic representation of NPM-ALK fusion tyrosine kinase. The fusion junction that the siRNA was designed to target is indicated in the upper panel. The lower panel shows the predicted short hairpin transcript with a $3^{\prime}-U_{5}$ pol III stop signal. (F) NPM-ALK-specific siRNA (NAsiRNA) sensitizes Ba/F3 cells transformed with NPM-ALK (N/A) to rapamycin in the cell viability assay. (G) Decreased expression of NPM-ALK and phosphorylation of p70 S6 kinase by stable expression of NAsiRNA. Ba/F3 cells transduced with empty retroviral vector were included as a control. 
A

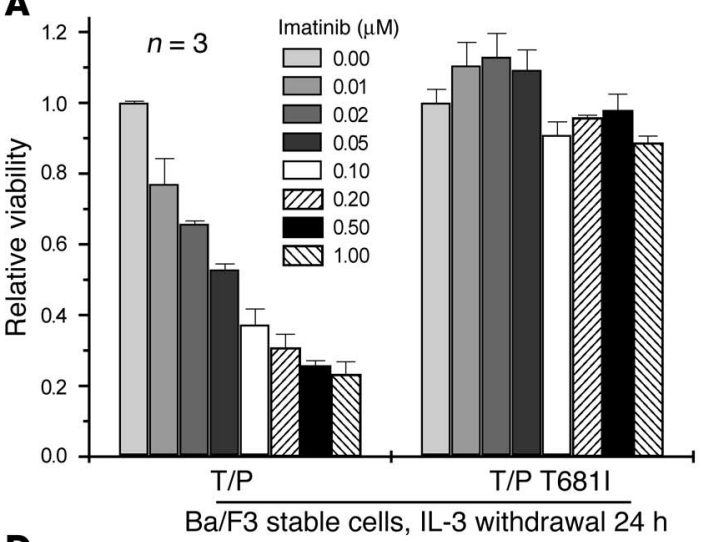

D

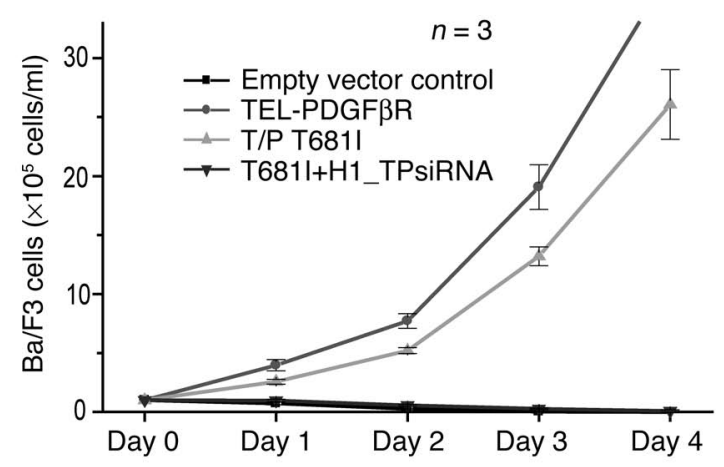

B

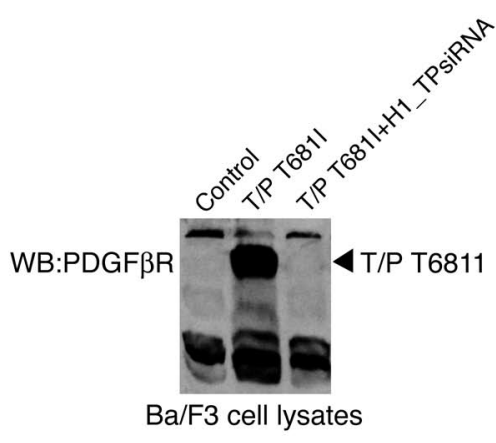

E

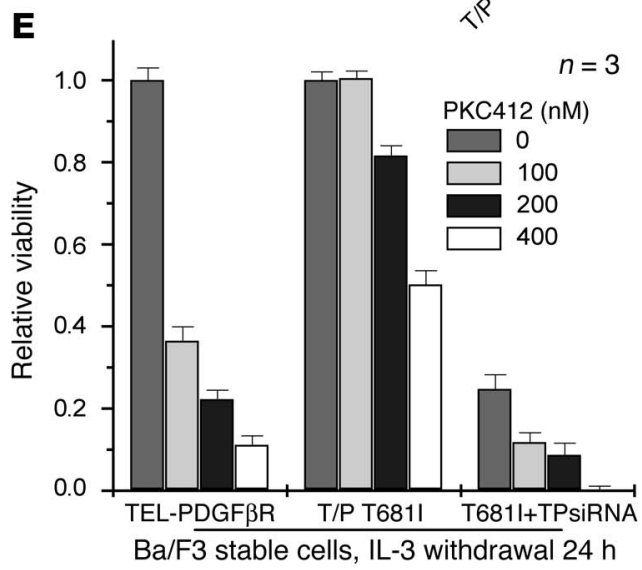

Figure 4

Stable expression of siRNA overcomes drug resistance. (A) TEL-PDGF $\beta$ R T681I is resistant to imatinib in the cell viability assay. (B) Expression of TPsiRNA decreases TEL-PDGF $\beta$ R T681I protein expression in stably transformed Ba/F3 cells. Western blot analysis was performed as described in Figure 1C. (C) Decreased proliferation of TEL-PDGF $\beta R$ T681I-transformed Ba/F3 cells by TPsiRNA in the cell viability assay. Cells were treated with IL-3 withdrawal for 24 hours. (D) Test for IL-3 independence of Ba/F3-derivative stable cell lines expressing TEL-PDGF $\beta R$ T681I with or without TPsiRNA. (E) TPsiRNA expression sensitizes TEL-PDGF $\beta R$ T681I-transformed Ba/F3 cells to inhibition by PKC412. The percentage of cell growth was normalized to the growth of cells in the absence of drug treatment.

activity of TEL-PDGF $\beta$ R (Figure 3B), which correlated with the nearly full reversion to IL-3 dependence of the growth of transformed cells with the same treatments (Figure 3A). These data provide the signaling basis for a mechanism by which suppression of TEL-PDGF $\beta R$ expression by siRNA sensitizes transformed cells to imatinib.

We also tested the effect of the mammalian target of rapamycin (mTOR) inhibitor rapamycin (25) on TEL-PDGF $\beta$ R-transformed cells in the presence or absence of TPsiRNA. mTOR is a serine/threonine kinase, and the IGFR-PI3K-Akt-mTOR signaling pathway is crucial for cell growth and survival (26). As a surrogate for mTOR activation, we evaluated one of the mTOR substrates, p70 S6 kinase (27), which is phosphorylated and activated in TEL-PDGF $\beta$ R-transformed cells (Figure 3D). Rapamycin alone was a potent inhibitor of cell growth in TEL-PDGF $\beta$ R-transformed Ba/F3 cells (Figure 3C). Furthermore, rapamycin in combination with TPsiRNA expression was more effective than either agent alone in the inhibition of TEL-PDGF $\beta R$-transformed Ba/F3 cells (Figure 3C). Similar results were obtained in silencing fusion tyrosine kinase NPM-ALK, which is associated with human anaplastic large-cell lymphoma (ALCL) (28), by the stable expression of fusion junction-specific siRNA and the addition of rapamycin (Figure 3, E-G), indicating that the strategy can be extrapolated to other oncogenic fusion tyrosine kinases.
Collectively, these data indicate that expression of siRNA targeting fusion tyrosine kinases can enhance the efficacy of cell killing in combination with inhibitors of the tyrosine kinases themselves or their downstream effectors.

We also investigated the potential use of inhibitory siRNA as a strategy to inhibit imatinib-resistant mutants of TEL-PDGF $\beta$ R. A potential imatinib-resistant mutation T681I was introduced into TEL-PDGF $\beta$ R. The T681I mutation in the context of PDGF $\beta R$ corresponds to the T315I mutation in $\mathrm{ABL}$, a common resistance mutation in $\mathrm{BCR}-\mathrm{ABL}^{+} \mathrm{CML}$ patients who relapse while on imatinib therapy $(6,7)$. Similarly, TEL-PDGF $\beta R$ T681I mutant showed resistance to imatinib. The IL-3-independent proliferation of Ba/F3 cells transduced with TEL-PDGF $\beta R$ T681I was not inhibited at concentrations of imatinib as high as $1.0 \mu \mathrm{M}$ (Figure $4 \mathrm{~A}$ ). Consistent with our previous results, stable expression of TPsiRNA not only decreased TEL-PDGF $\beta$ R T681I protein expression (Figure $4 \mathrm{~B}$ ), but resulted in a significantly slower proliferative rate of TEL-PDGF $\beta$ R T681I-transduced Ba/F3 cells compared with cells expressing either TEL-PDGF $\beta$ R wild type or T681I mutant alone (Figure 4, C and D). The majority of cells coexpressing TELPDGFßR T681I and TPsiRNA underwent apoptosis and died by day 4 in the absence of IL-3 (Figure 4D). 
In addition, we found that the imatinib-resistant TEL-PDGF $\beta R$ T681I mutant was efficiently inhibited by an alternative small molecule inhibitor PKC412 (Figure 4E). PKC412 is an inhibitor of FMS-like receptor tyrosine kinase 3 (FLT3), PKC, kinase insert domain receptor (KDR) c-KIT, PDGFR $\alpha$ and PDGF $\beta R$ (29-31), and this agent is currently being evaluated as molecularly-targeted therapy in a phase II clinical trial for AML patients with FLT3 activating mutations (32). Moreover, the dose-response curves demonstrated that, although TEL-PDGF $\beta$ R T681I-transformed Ba/F3 cells were not sensitized to imatinib by coexpression of TPsiRNA (data not shown), they were sensitized to PKC412 (Figure 4E). The $\mathrm{IC}_{50}$ of TEL-PDGF $\beta R$ T681I-transformed cells for PKC412 is approximately $400 \mathrm{nM}$, which is decreased to approximately $100 \mathrm{nM}$ by coexpression of TPsiRNA (Figure 4E). Taken together, these data demonstrate that the expression of siRNA not only enhances the efficacy of inhibition by small molecule inhibitors of susceptible tyrosine kinases but may also be useful for the inhibition of mutant tyrosine kinases that are resistant to small molecule inhibitors.

\section{Discussion}

In this study, we present a retroviral delivery system for stable expression of siRNA directed against the unique fusion junction sequence of TEL-PDGF $\beta$ R in transformed hematopoietic cells. This retrovirus-based method allows for stable expression of siRNA in mammalian cells and overcomes the problems of poor transfection efficiency of small RNAs into many hematopoietic cell lines. Moreover, because the provirus containing siRNA expression cassette is integrated into the genome of the stably transduced cells, this system also provides a useful tool for monitoring the long-term effects of stably expressing siRNA in hematopoietic cells.

Stable expression of the siRNA markedly inhibits TEL-PDGF $\beta R$ expression and activation of its downstream signaling effectors. In addition, our results showing inhibition of NPM-ALK by using siRNA directed to the fusion junction indicate that this approach may be broadly applicable for the inhibition of oncogenic fusion proteins. Although the TEL-PDGF $\beta$ R protein level is markedly reduced by expression of TEL-PDGF $\beta$ R-specific siRNA (TPsiR$\mathrm{NA}$ ), there is a residual low level of target protein expression. Our results indicate that expression of siRNA alone is effective, but not sufficient, to completely abrogate TEL-PDGF $\beta$ R-induced transformation in cell culture and murine allograft models. However, our data demonstrate that stable expression of siRNA sensitizes TELPDGF $\beta R$-transformed cells to the small molecule inhibitors imatinib and rapamycin. These findings indicate that siRNA directed to the unique fusion junction sequences of the target fusion tyrosine kinases can enhance the efficacy of cell killing by other molecularly targeted therapies, such as direct inhibitors of the tyrosine kinases themselves or indirect inhibitors of their downstream signaling pathways. It is possible to enhance the siRNA inhibitory effect by designing vectors that contain multiple copies of siRNA expression cassettes or by superinfection strategies.

Moreover, expression of TPsiRNA effectively inhibits an imatinib-resistant mutant of TEL-PDGF $\beta R$, indicating that this siRNA approach provides a strategy opportunity to overcome clinically identified drug resistance. In addition, the imatinibresistant TEL-PDGF $\beta$ R T681I mutant is efficiently inhibited by PKC412. In contrast to imatinib, which binds to and inhibits $\mathrm{ABL}$ by trapping the kinase in an inactive conformation (33, 34), PKC412 is a derivative of staurosporine and may inhibit the target kinases by binding within the ATP-binding pocket of the active conformation $(35,36)$, thus inhibiting the TEL-PDGF $\beta R$ imatinib-resistant mutant. Indeed, we reported previously that PKC412 effectively inhibits an imatinib-resistant fusion tyrosine kinase FIP1L1-PDGFR $\alpha$ mutant T674I, analogous to the BCRABL T315I mutant (37). Here we show that the stable expression of TPsiRNA enhances PKC412-mediated killing of TEL-PDGF $\beta$ R T681I-transformed cells. These findings indicate the feasibility of a combination strategy to overcome drug resistance.

In conclusion, this retroviral system for the delivery and stable expression of siRNA, as well as the strategy to design sequence-specific siRNA targeting the fusion junctions can be used to specifically inhibit chimeric oncogenic genes that are expressed as a consequence of aberrant chromosomal translocations such as BCR-ABL and TEL- PDGF $\beta R$, or deletions such as FIP1L1-PDGFR $\alpha(1,38)$. Targeting the fusion junction may decrease the likelihood of nonspecific toxicities due to inhibition of the respective wild-type alleles of the fusion genes. Most small molecule inhibitors, including imatinib, are selective rather than specific. This may be an advantage in that leukemias and certain solid tumors associated with mutational activation of ABL, PDGF $\beta$ R, PDGFR $\alpha$ or KIT may be effectively treated with a single agent, such as imatinib. However, it is also possible that a broad range of specificity could contribute to the toxicities of small molecule kinase inhibitors when used either alone or in combination with other agents. In addition, it is clear that, although drugs like imatinib are remarkably effective, they are disease-remitting agents rather than curative, and that clinical resistance develops to these drugs when they are used as single agents (2, 6-9). Finally, although small molecule inhibitors have shown great promise for the treatment of cancer, there are no small molecule inhibitors currently available for clinical use for a number of fusion kinases, including NPM-ALK (28) and ZNF198-FGFR1 (39). For these reasons, alternative molecularly targeted therapies, including the application of inhibitory siRNA, are under development in a variety of contexts that might enhance specificity and overcome the problem of resistance. Although the efficient delivery of siRNA to target cells will be a challenging problem, our data indicate that the use of siRNA in combination with other molecularly targeted therapies may have therapeutic value.

\section{Acknowledgments}

We gratefully acknowledge a critical reading of the manuscript by David Sternberg, administrative assistance from Alexis Bywater, and valuable discussion with members of the Gilliland laboratory. This work was supported in part by NIH grants CA676996 and DK50654, the Leukemia and Lymphoma Society, and a Dana-Farber/Harvard Cancer Center New Nodal Interaction Grant. N.R. Wall is supported by National Research Service Award F32 CA097802 from the Cancer Training Branch/ National Cancer Institute. Y. Shi is supported by NIH grant R01GM53874. J. Chen is a Fellow of the Leukemia and Lymphoma Society, and D.G. Gilliland is an investigator of the Howard Hughes Medical Institute.

Received for publication December 1, 2003, and accepted in revised form March 30, 2004.

Address correspondence to: D. Gary Gilliland, Division of Hematology, Brigham and Women's Hospital, 75 Francis Street, Boston, Massachusetts 02115, USA. Phone: (617) 355-9092; Fax: (617) 355-9093; E-mail: ggilliland@rics.bwh.harvard.edu. 
1. Dash, A., and Gilliland, D.G. 2001. Molecular genetics of acute myeloid leukaemia. Best Pract. Res. Clin. Haematol. 14:49-64.

2. Capdeville, R., Buchdunger, E., Zimmermann, J., and Matter, A. 2002. Glivec (STI571, imatinib), a rationally developed, targeted anticancer drug. Nat. Rev. Drug Discov. 1:493-502.

3. Druker, B.J. 2003. Imatinib alone and in combination for chronic myeloid leukemia. Semin. Hematol. 40:50-58.

4. Demetri, G.D., et al. 2002. Efficacy and safety of imatinib mesylate in advanced gastrointestinal stromal tumors. N. Engl. J. Med. 347:472-480.

5. Apperley, J.F., et al. 2002. Response to imatinib mesylate in patients with chronic myeloproliferative diseases with rearrangements of the plateletderived growth factor receptor beta. N. Engl. J. Med. 347:481-487.

6. Shah, N.P., et al. 2002. Multiple BCR-ABL kinase domain mutations confer polyclonal resistance to the tyrosine kinase inhibitor imatinib (STI571) in chronic phase and blast crisis chronic myeloid leukemia. Cancer Cell. 2:117-125.

7. Gorre, M.E., et al. 2001. Clinical resistance to STI571 cancer therapy caused by BCR-ABL gene mutation or amplification. Science. 293:876-880.

8. Druker, B.J., et al. 2001. Efficacy and safety of a specific inhibitor of the BCR-ABL tyrosine kinase in chronic myeloid leukemia. N. Engl. J. Med. 344:1031-1037.

9. Druker, B.J., et al. 2001. Activity of a specific inhibitor of the BCR-ABL tyrosine kinase in the blast crisis of chronic myeloid leukemia and acute lymphoblastic leukemia with the Philadelphia chromosome. N. Engl. J. Med. 344:1038-1042.

10. Elbashir, S.M., Lendeckel, W., and Tuschl, T. 2001 RNA interference is mediated by 21 - and 22 -nucleotide RNAs. Genes Dev. 15:188-200.

11. Elbashir, S.M., et al. 2001. Duplexes of 21-nucleotide RNAs mediate RNA interference in cultured mammalian cells. Nature. 411:494-498.

12. Sharp, P.A. 2001. RNA interference - 2001. Genes Dev. 15:485-490.

13. Wilda, M., Fuchs, U., Wossmann, W., and Borkhardt, A. 2002. Killing of leukemic cells with a $\mathrm{BCR} / \mathrm{ABL}$ fusion gene by RNA interference (RNAi). Oncogene. 21:5716-5724.

14. Wohlbold, L., et al. 2003. Inhibition of bcr-abl gene expression by small interfering RNA sensitizes for imatinib mesylate (STI571). Blood. 102:2236-2239.

15. Scherr, M., et al. 2003. Specific inhibition of bcr-abl gene expression by small interfering RNA. Blood. 101:1566-1569.

16. Paul, C.P., Good, P.D., Winer, I., and Engelke, D.R. 2002. Effective expression of small interfering RNA in human cells. Nat. Biotechnol. 20:505-508.

17. Miyagishi, M., and Taira, K. 2002. U6 promoterdriven siRNAs with four uridine $3^{\prime}$ overhangs efficiently suppress targeted gene expression in mammalian cells. Nat. Biotechnol. 20:497-500.

18. Lee, N.S., et al. 2002. Expression of small interfering RNAs targeted against HIV-1 rev transcripts in human cells. Nat. Biotechnol. 20:500-505.

19. Sui, G., et al. 2002. A DNA vector-based RNAi technology to suppress gene expression in mammalian cells. Proc. Natl. Acad. Sci. U. S. A. 99:5515-5520.

20. Golub, T.R., Barker, G.F., Lovett, M., and Gilliland, D.G. 1994. Fusion of PDGF receptor beta to a novel ets-like gene, tel, in chronic myelomonocytic leukemia with $\mathrm{t}(5 ; 12)$ chromosomal translocation. Cell. 77:307-316.

21. Carroll, M., et al. 1997. CGP 57148, a tyrosine kinase inhibitor, inhibits the growth of cells expressing BCR-ABL, TEL-ABL, and TEL-PDGFR fusion proteins. Blood. 90:4947-4952.

22. Brummelkamp, T.R., Bernards, R., and Agami, R. 2002. A system for stable expression of short interfering RNAs in mammalian cells. Science. 296:550-553.

23. Barton, G.M., and Medzhitov, R. 2002. Retroviral delivery of small interfering RNA into primary cells. Proc. Natl. Acad. Sci. U. S. A. 99:14943-14945.

24. Tomasson, M.H., et al. 2000. Fatal myeloproliferation, induced in mice by TEL/PDGF $\beta$ R expression, depends on PDGF $\beta$ R tyrosines 579/581. J. Clin. Invest. 105:423-432.

25. Sabers, C.J., et al. 1995. Isolation of a protein target of the FKBP12-rapamycin complex in mammalian cells. J. Biol. Chem. 270:815-822.

26. Huang, S., and Houghton, P.J. 2003. Targeting mTOR signaling for cancer therapy. Curr. Opin. Pharmacol. 3:371-377.

27. Burnett, P.E., Barrow, R.K., Cohen, N.A., Snyder, S.H., and Sabatini, D.M. 1998. RAFT1 phosphorylation of the translational regulators p70 S6 kinase and 4EBP1. Proc. Natl. Acad. Sci. U. S. A. 95:1432-1437.
28. Ladanyi, M. 1997. The NPM/ALK gene fusion in the pathogenesis of anaplastic large cell lymphoma. Cancer Surv. 30:59-75.

29. Fabbro, D., et al. 2000. PKC412 - a protein kinase inhibitor with a broad therapeutic potential. Anticancer Drug Des. 15:17-28.

30. Andrejauskas-Buchdunger, E., and Regenass, U. 1992. Differential inhibition of the epidermal growth factor-, platelet-derived growth factor-, and protein kinase $\mathrm{C}$-mediated signal transduction pathways by the staurosporine derivative CGP 41251. Cancer Res. 52:5353-5358.

31. Weisberg, E., et al. 2002. Inhibition of mutant FLT3 receptors in leukemia cells by the small molecule tyrosine kinase inhibitor PKC412. Cancer Cell. 1:433-443.

32. Stone, R.M., et al. 2003. PKC412, an oral FLT3 inhibitor, has activity in mutant FLT3 acute myeloid leukemia (AML): a phase II clinical trial [abstract]. Blood. 100:86a.

33. Nagar, B., et al. 2002. Crystal structures of the kinase domain of c-Abl in complex with the small molecule inhibitors PD173955 and imatinib (STI571). Cancer Res. 62:4236-4243.

34. Schindler, T., et al. 2000. Structural mechanism for STI-571 inhibition of abelson tyrosine kinase. Science. 289:1938-1942.

35. Toledo, L.M., and Lydon, N.B. 1997. Structures of staurosporine bound to CDK2 and CAPK - new tools for structure-based design of protein kinase inhibitors. Structure. 5:1551-1556.

36. Lamers, M.B., Antson, A.A., Hubbard, R.E., Scott, R.K., and Williams, D.H. 1999. Structure of the protein tyrosine kinase domain of C-terminal Src kinase (CSK) in complex with staurosporine. J. Mol. Biol. 285:713-725.

37. Cools, J., et al. 2003. PKC412 overcomes resistance to imatinib in a murine model of FIP1L1PDGFRalpha-induced myeloproliferative disease. Cancer Cell. 3:459-469.

38. Cools, J., et al. 2003. A tyrosine kinase created by fusion of the PDGFRA and FIP1L1 genes as a therapeutic target of imatinib in idiopathic hypereosinophilic syndrome. N. Engl. J. Med. 348:1201-1214.

39. Xiao, S., et al. 1998. FGFR1 is fused with a novel zinc-finger gene, ZNF198, in the $\mathrm{t}(8 ; 13)$ leukaemia/lymphoma syndrome. Nat. Genet. 18:84-87. 